\title{
Achievement of sustained deep remission with adalimumab in a patient with both refractory ulcerative colitis and seronegative erosive rheumatoid arthritis
}

\author{
G. Andrisani', E. Gremese ${ }^{2}$, L. Guidi' ${ }^{1}$ A. Papa ${ }^{1}$, M. Marzo', \\ C. Felice ${ }^{1}$, D. Pugliese ${ }^{1}$, A. Armuzzi ${ }^{1}$ \\ 1'Internal Medicine and Gastroenterology Unit, Complesso Integrato Columbus, Catholic University; \\ ${ }^{2}$ Rheumatology Unit, Complesso Integrato Columbus, Catholic University, Rome, Italy
}

\begin{abstract}
SUMMARY
Inflammatory bowel disease (IBD) is commonly associated with peripheral inflammatory arthritis, and it has been estimated that as many as $12 \%$ of IBD patients report these manifestations. However, rheumatoid arthritis (RA) is rarely associated with ulcerative colitis (UC). Among all the biological agents available, nine have been currently approved for the treatment of RA. Conversely, only Infliximab and recently Adalimumab have been approved for UC. In particular, the efficacy of Adalimumab in UC has been demonstrated by both recent randomized controlled trials and real-life studies. Moreover, Adalimumab is a well-established treatment for RA. Herein, we describe a patient with RA and UC treated successfully with ADA.
\end{abstract}

Key words: Ulcerative colitis, Rheumatoid arthritis, Adalimumab.

Reumatismo, 2013; 65 (2): 75-78

\section{INTRODUCTION}

Tnflammatory bowel disease (IBD) is commonly associated with peripheral inflammatory arthritis, and it has been estimated that as many as $12 \%$ of IBD patients report these manifestations (1). Peripheral arthritis associated with ulcerative colitis (UC) is oligoarticular and asymmetric, affecting predominantly the lower extremity joints and it is always seronegative (2). This association may be due to a similar autoimmune pathogenesis of the two diseases.

In fact, almost all the immune-mediated diseases are more common among IBDpatients, compared to non-IBD ones.

However, rheumatoid arthritis (RA) is rarely associated with $\mathrm{UC}$, maybe because differential diagnosis of RA becomes quite challenging.

To the best of our knowledge, only a few of these cases have been reported in the literature (3-10) and none regarding anti-TNF treatment of the two diseases. Herein, we describe a RA patient that was diagnosed with UC and successfully treated with adalimumab (ADA).

\section{CASE REPORT}

Here we report the case of a 54-year-old woman who was admitted to our Unit for abdominal pain, fever, rectal bleeding and diffuse arthralgia. She was diagnosed with seronegative RA since the age of 37 , on the basis of peripheral (hand and feet) symmetric polyarthritis, according to 1987 the American College of Rheumatology criteria (11). She was previously treated with long-term steroid therapy and methotrexate (discontinued for disease remission), and then with hydroxichloroquine $(6 \mathrm{mg} /$ $\mathrm{kg}$ /day) until the admission at our unit, with sufficient control of RA disease. Six months before admission (March 2007) she had been diagnosed with extensive ulcerative colitis according to clinical symptoms and endoscopic/histological findings. Ileocolonoscopy showed normal terminal
Corresponding author: Dr. Alessandro Armuzzi Internal Medicine and Gastroenterology Unit Complesso Integrato Columbus

Università Cattolica del Sacro Cuore Via Moscati, 31-33 - 00168 Roma, Italy E-mail: alearmuzzi@yahoo.com 
ileum and diffuse erosions and friability of the mucosa, which was spontaneously bleeding over the entire colonic tract. Multiple biopsies revealed distortion of gland architecture, mucin depletion, marked inflammatory infiltration in the lamina propria and cryptitis. Consistent with this diagnosis, abdominal ultrasonography depicted mild parietal thickening of the colon and normal thickness of the ileum. She was started on treatment with oral and topical mesalamine after diagnosis and then received one course of oral corticosteroids with partial improvement of symptoms, which relapsed during tapering.

In September 2007, the severity of UCrelated symptoms ( 8 bowel movements/ day with blood and mild fever) led to admission to our Unit. She presented also peripheral arthralgias. Physical examination revealed tachycardia and tenderness in the left abdominal quadrants with no rebound or guarding. Abdominal $\mathrm{X}$ ray did not show free air, air fluid levels, colonic or small bowel dilation.

Main laboratory findings revealed moderate anemia (hemoglobin: $10.7 \mathrm{~g} / \mathrm{dL}$ ), increase of C-reactive protein level of (34.8 $\mathrm{mg} / \mathrm{L}$ ) and erythrocyte sedimentation rate (ESR) $(42 \mathrm{~mm} / \mathrm{h})$, low serum albumin levels $(3.2 \mathrm{~g} / \mathrm{dL})$. Stool colture and $C$. difficile toxin tests were negative. On the basis of clinical and biochemical features, the patient was administered intravenous prednisolone $(1 \mathrm{mg} / \mathrm{kg} /$ day $)$ for seven days, but no significant benefit was observed. Therefore, anti-TNF treatment with infliximab $(5 \mathrm{mg} / \mathrm{kg})$ and azathioprine $(2 \mathrm{mg} / \mathrm{kg})$ was started and steroid then tapered. After three months, azathioprine was stopped due to an increase in liver enzyme levels. At that time the patient was experiencing relief of gastrointestinal symptoms, but persistence of arthralgia, after an initial improvement. In April 2008, a relapse of bowel symptoms required another oral steroid cycle. A few months later (June 2008) the patient developed an infusion reaction to infliximab (skin rash and dyspnea) and, therefore, the drug was discontinued. She underwent ileocolonoscopy that showed purulent mucous secretions, dyschromic and

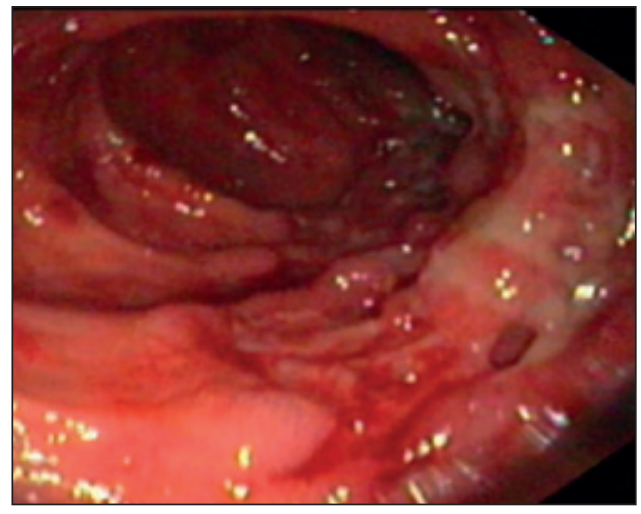

Figure 1 - Several erosions and ulcers in sigmoid colon.

hyperemic mucosa in the right and transverse colon, swollen ileo-caecal valve and several erosions and ulcers in sigmoid colon and rectum (Fig. 1). Furthermore, she was evaluated by a rheumatologist for the persistence of joint symptoms. The patient presented 12 swollen and 15 tender joints with a disease activity index on 28 joints (DAS28) of 6.8, indicating a high disease activity. Hand and feet X-rays showed characteristics RA joint erosions. No sacroileitis was found and the lumbar spine was normal. The rheumatologist recommended methotrexate (15 mg/week), folic acid and methylprednisolone (16 mg/day), with only partial improvement.

Based on the intestinal symptoms and endoscopic findings observed on July 2008, the patient started adalimumab (ADA) subcutaneously, at the dosage of $160 / 80 \mathrm{mg}$ as induction and then $40 \mathrm{mg}$ every two weeks as maintenance, with considerable clinical improvement already after induction treatment (hemoglobin $12.6 \mathrm{~g} / \mathrm{dL}, \mathrm{C}$-reactive protein $2.2 \mathrm{mg} / \mathrm{L}$, ESR $10 \mathrm{~mm} / \mathrm{h}$ ). One year later, she underwent colonoscopy that showed diffuse hyperemia and decrease of vascular pattern. Multiple biopsies revealed mild chronic inflammatory infiltrate in the lamina propria and mild glandular atrophy, suggesting a condition of quiescent UC. The rheumatology evaluation was consistent with disease in remission (no swollen joints, 2 tender joints, DAS28 2.54). In view of the endoscopic picture and good clinical condition of the patient 


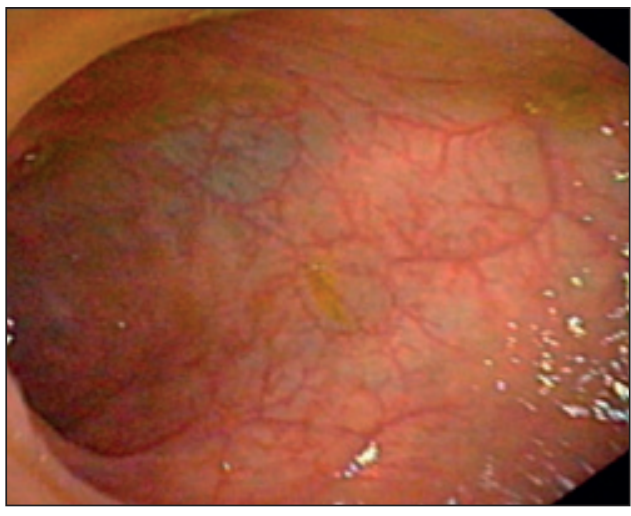

Figure 2 - Mucosal healing.

it was decided to continue treatment with ADA along with low doses of methotrexate $(10 \mathrm{mg} /$ week $)$ and no steroid therapy. In December 2009, in according to rheumatologist, the patient discontinued methotrexate due to persistent RA remission. In August 2011, after 3 years of therapy with ADA, another colonoscopy was performed and the mucosa appeared healed throughout the entire colon (Fig. 2).

\section{DISCUSSION}

Among all the biological agents available, nine have been currently approved for the treatment of RA. Conversely, only two antitumor necrosis factor alpha (anti-TNF- $\alpha$ ) agents, namely Infliximab and recently ADA, have been approved for for the treatment of UC. In particular, the efficacy of ADA in UC has been demonstrated by both recent randomized controlled trials and real-life studies (12-16). Moreover, ADA is a well-established treatment for RA.

The described patient suffered from ulcerative pancolitis occurring during the course of a long-term history of RA, and was successfully treated with ADA after failure of other available drugs.

Even if we consider only the gastrointestinal disease, the clinical features of this patient are quite difficult to manage from a medical point of view. In fact, the use of pharmacological therapies is limited and in cases of refractoriness the only other option for this patient is colectomy. Surgery is theoretically curative for UC but there are many possible post-operative complications and, even in the best outcome, the patient will have 4/5 bowel movements per day.

Moreover, in our case we have a complicating factor that is the association of RA. To date, no specific trials aiming to evaluate anti-TNF- $\alpha$ treatment in patients with RA and UC have been performed. To our knowledge, few cases of RA with concomitant UC have been reported, but none regarding a successful treatment with ADA (3-10). Some of these cases evaluate possible pathogenetic mechanism for the concomitance of the two diseases (3$5)$. One recent report highlights the further association of selective immunoglobulin A deficiency in a patient with long-standing RA, which developed UC (7). Sugisaki et $a l$. describes a patient that was quite similar to the present case, but was successfully treated with mesalamine enema (6).

A further three reports describe treatment using a biological agent, but not specifically with anti TNF- $\alpha$ to treat the two associated pathologies. Interestingly, two other studies reported the occurrence of UC a few months after treatment with abatacept (during a clinical trial) in patients with RA (8) and the onset of UC during the biologic treatment for RA currently used also to treat UC (9), respectively. Finally, a report by Mayer et al, describes a complex case of many overlapping syndromes: rheumatoid arthritis, ulcerative colitis, spondyloarthritis and systemic lupus erythematosus. However, in that case, ADA was introduced only after an urgent colectomy, performed for multidrug-resistant ulcerative colitis complicated with perforation, to treat arthritis (10).

In the current case, rheumatoid arthritis started many years before ulcerative colitis, and, until the onset of the intestinal disease, had a relatively benign course (with disease remission and discontinuation of therapy), although with the development of typical $\mathrm{x}$-rays erosions, despite the seronegativity. After ulcerative colitis onset, rheumatoid arthritis seems to have had an accelerated course and a severe flare that does not re- 
spond even to the first anti-TNF $\alpha$ therapy used, suggesting that the underlying mechanisms of inflammation in the two diseases are common. The use of adalimumab, also in presence of rheumatoid arthritis negative prognostic factors (i.e. high disease activity -DAS28 6.4-, x-rays erosions, long disease duration, non-response to the first antiTNF), appears to be very effective.

In conclusion, considering the scarce data available so far, our case suggests that ADA can have a beneficial effect in patients with both ulcerative colitis and rheumatoid arthritis, refractory to conventional therapy. Furthermore, we also achieved mucosal healing that is, to date, one of the most ambitious goals in a patient with IBD. Future studies, including randomized clinical trials are needed to confirm our findings.

Conflict of interests: AA received consultancy from AbbVie and MSD; lecture fees from AbbVie, MSD, Chiesi, Ferring, Nycomed, and Otsuka; and educational grants from AbbVie and MSD. LG received educational grants from AbbVie, MSD. The authors report no other conflicts of interest.

Contributions: all authors contributed equally.

\section{REFERENCES}

1. Palm $\varnothing$, Moum B, Jahnsen J, Gran JT. The prevalence and incidence of peripheral arthritis in patients with inflammatory bowel disease, a prospective population-based study (the IBSEN study). Rheumatol (Oxford). 2001; 40: 1256-61.

2. Scarpa R, del Puente A, D'Arienzo A, et al. The arthritis of ulcerative colitis: clinical and genetic aspects. J Rheumatol. 1992: 19: 373-7.

3. Klausen T, Amris K, Helin P. Ulcerative colitis complicating seronegative HLA-A2-B27 rheumatoid arthritis with sacroiliitis. Ann Rheumat Dis. 1992; 51: 1009-11.

4. Adachi Y, Hinoda Y, Takashi H, et al. Rheumatoid arthritis associated with ulcerative colitis. J Gastroenterol. 1996; 31: 590-5.
5. Aydin Y, Ozçakar L, Yildiz M, Akinci A. Liaison between rheumatoid arthritis and ulcerative colitis. Rheumatol Int. 2003; 23: 47-48.

6. Sugisaki K, Honma F, Iwadate H, et al. Ulcerative colitis occurring in the course of rheumatoid arthritis: a case successfully treated with mesalamine enema. Int Med. 2004; 43: 1046-50.

7. Asada Y, Isomoto H, Shikuwa S, et al. Development of ulcerative colitis during the course of rheumatoid arthritis: association with selective IgA deficiency. World J Gastroenterol. 2006; 12: 5240-3.

8. Amezcua-Guerra LM, Hernández-Martínez B, Pineda C, Bojalil R. Ulcerative colitis during CTLA-4Ig therapy in a patient with rheumatoid arthritis. Gut. 2006; 55: 1059-60.

9. Rosu A, Ciurea P, Musetescu A. Ulcerative colitis developed during the course of rheumatoid arthritis treated with infliximab: a case report. Clin Exp Rheumatol. 2008; 26: AbsR4.

10. Mayer M, Baresic M, Anic B, et al. Complex case of overlap syndrom (RA, SLE, SPA, UC). 7th Biennial International Congress on Autoimmunity, May 5-10, 2010, Ljubljana, Slovakia. Abstract 1029.

11. Arnett FC, Edworthy SM, Bloch DA, et al. The American Rheumatism Association 1987 revised criteria for the classification of rheumatoid arthritis. Arthritis Rheum. 1988; 31: 315-24.

12. Reinisch W, Sandborn WJ, Hommes DW, et al. Adalimumab for induction of clinical remission in moderately to severely active ulcerative colitis: results of a randomised controlled trial. Gut. 2011; 60: 780-7.

13. Sandborn WJ, Van Assche G, Reinisch W, et al. Adalimumab induces and maintains clinical remission in patients with moderate-tosevere ulcerative colitis. Gastroenterology. 2012; 142: 257-65.e1-3.

14. Gies N, Kroeker KI, Wong K, Fedorak RN. Treatment of ulcerative colitis with adalimumab or infliximab: long-term follow-up of a single-centre cohort. Aliment Pharmacol Ther. 2010; 32: 522-8.

15. Taxonera C, Estelles J, Blanco I, et al. Adalimumab induction and maintenance therapy for patients with ulcerative colitis previously treated with infliximab. Aliment Pharmacol Ther. 2011; 33: 340-8.

16. Armuzzi A, Biancone L, Daperno M, et al. Adalimumab in active ulcerative colitis: a "real-life" observational study. Dig Liver Dis. In press 2013. 